\title{
Desencadenantes de la lealtad de padres de familia hacia un programa deportivo para sus hijos. Avances en el campo de la gestión deportiva
}

\author{
Triggers of Family LoyaltyTowards a Sports Program for your Children. \\ Advances in the field of sports management
}

Recibido: 02-04-2019 • Aprobado: 04-06-2019 • Página inicial: 165 - Página final: 181

Andrés Leonardo Colorado Arango*

\begin{abstract}
Resumen: el presente trabajo tuvo como objetivo identificar factores que desencadenan la lealtad de los padres de familia hacia un programa de iniciación deportiva en el que participan sus hijos, a partir de la aplicación de una encuesta estructurada a los clientes de un centro deportivo en la ciudad de Medellín, Colombia. Los resultados fueron analizados en aspectos de fiabilidad y validez siguiendo un análisis factorial confirmatorio, en el cual se obtuvieron adecuados indicadores de bondad de ajuste, además se demostraron las relaciones entre las variables utilizando un modelo de ecuaciones estructurales que derivó en la aceptación de la mayoría de las hipótesis y la comprobación de la pertinencia de la Teoría del Comportamiento Planificado (TPB) para explicar las variables que desencadenan la lealtad de los padres de familia a los servicios deportivos que ofrece la institución analizada.
\end{abstract}

Palabras clave: lealtad, servicios deportivos, Teoría del Comportamiento Planificado.

\begin{abstract}
The objective of this work was to identify factors that trigger the loyalty of parents to a sports initiation program in which their children participate, based on the application of a structured survey the clients of a sports center in the city of Medellín - Colombia. The results were analyzed in aspects of reliability and validity following a confi rmatory factorial analysis, in which adequate indicators of goodness of $f i t$ were obtained, in addition the relationships among the variables were demonstrated using a model of structural equations that led to the acceptance of most of the hypothesis and the verifi cation that Theory of Planned Behavior (TPB) is relevant to explain the variables that trigger the loyalty of parents to the sports services offered by the institution analyzed.
\end{abstract}

Keywords: Loyalty, Sports Services, Theory of Planned Behavior.

\footnotetext{
* Magister en Administración y Licenciado en Educación Física de la Universidad de Antioquia. Docente Tiempo Completo del Politécnico Colombiano Jaime Isaza Cadavid, Colombia y Miembro del Grupo de Investigación GESTAS de la misma Institución. Docente de cátedra del Instituto de Educación Física de la Universidad de Antioquia. andrescolorado@elpoli.edu.co

ORCID: https://orcid.org/0000-0001-7892-6733
} 


\section{Contexto colorado, A}

\section{Gatilhos da lealdade da família Rumo a um programa esportivo para seus filhos \\ Avanços no campo da gestão esportiva}

Resumo: o presente trabalho teve como objetivo de identificar fatores que desencadeiam a lealdade dos pais em relação a um programa de iniciação esportiva em que seus filhos participam, a partir da aplicação de uma pesquisa estruturada de clientes de um centro esportivo na cidade de Medellín, Colômbia Os resultados foram analisados. em aspectos de confiabilidade e validade após uma análise fatorial confirmatória, na quais indicadores adequados foram obtidos boa qualidade de ajuste, eles também foram demonstrados os relacionamentos entre as variáveis usando um modelo de equações estruturais que resultou na aceitação da maioria das hipóteses e a verificação do relevância da teoria do comportamento Planejado (TPB) para explicar as variáveis que desencadeiam a lealdade dos pais de serviços de família a esportes oferecidos a instituição analisada.

Palavras-chave: lealdade, serviços desportivos, Teoria do Comportamento Planejado. 


\section{Introducción}

El deporte es un fenómeno social que trasciende las fronteras y las barreras idiomáticas, y es una de las actividades humanas con mayor aceptación y diversificación en el mundo así como los valores que trae consigo: el buen estado físico, el juego limpio, el trabajo en equipo y la búsqueda de excelencia, que son universales (Naciones Unidas, 2005). El deporte moderno no solo ha llevado diversión y excelencia física, el comercio ha aprovechado su alcance para permear a deportistas, aficionados y dirigentes, tanto que, en la actualidad el Consejo Superior de Deportes de España estima en dieciocho mil millones de euros el volumen económico que mueve el deporte en ese país. Instalaciones deportivas, material deportivo, profesionales directos, puestos de trabajo relacionados con la restauración, construcción, turismo y merchandising, y una larga serie de actividades que año tras año van engrosando la actividad económica que genera el deporte en todas sus manifestaciones, con cifras que dan cuenta de la dimensión de este negocio a nivel mundial.

Como consecuencia de ese fenómeno, los programas de iniciación deportiva a temprana edad, y específicamente en el fútbol, se han desarrollado basados en la demanda de padres de familia que pretenden acelerar el proceso de formación de deportistas por medio de programas serios y estructurados, ya que el desarrollo de sus hijos está en juego, lo que obliga a las organizaciones deportivas a generar ofertas que se adapten a los diversos intereses y posibilidades de los clientes.

Desde el sector público y privado se realizan campañas con el objetivo de fidelizar a los padres de familia a programas deportivos para sus hijos, sin embargo, se desconocen los factores que influyen en el individuo para desarrollar dicho comportamiento. Estar al tanto de los antecedentes del comportamiento de los clientes permitiría a las organizaciones enfocar sus campañas de comunicación y el diseño de su portafolio de servicios en elementos relevantes que influyan en la decisión del público objetivo.

Por lo anterior, es fundamental identificar los factores cognitivos, afectivos y de personalidad que inciden sobre la lealtad hacia un programa deportivo para niños, así como las interrelaciones entre dichos factores y su incidencia directa $\mathrm{o}$ indirecta sobre el comportamiento.

En relación con lo anterior, esta investigación tuvo como objetivo identificar los factores que desencadenan la lealtad de los clientes de una escuela de iniciación deportiva en la ciudad de Medellín, Colombia, mediante la aplicación de la 


\section{Contexto colorado, A.}

Teoría del Comportamiento Planificado. Para ello se construyó un marco teórico y un estado de arte del tema, posteriormente se propuso un modelo de hipótesis, al cual se le garantizó la validez y la fiabilidad mediante un análisis factorial confirmatorio y finalmente se contrastaron las hipótesis mediante ecuaciones estructurales.

\section{Marco teórico}

La Teoría del Comportamiento Planificado (TPB, por sus siglas en inglés) de Ajzen (1985) se ha aplicado como modelo para analizar diversas situaciones a partir de la valoración y ponderación de factores como "la actitud, la norma subjetiva y el control percibido" (Ajzen, 2010), aspectos decisivos para desencadenar la intención de materializar un comportamiento, por lo cual, áreas específicas de la administración como el marketing la utilizan regularmente para la modelación de estrategias.

La revisión de publicaciones de gran impacto internacional muestra que la TPB se ha utilizado como herramienta para comprender las decisiones de adopción de la tecnología y de inversión en ella (Lynne y Casey, 1995), para la valoración de estrategias que incentiven el consumo de frutas y verduras (Della, DeJoy y Lance, 2008), para comprender las variables en el campo del mercadeo para la comercialización de productos y servicios amigables con el ambiente (Ferdous, 2010), para identificar las actitudes de la población juvenil frente al mercadeo en teléfonos móviles (Yang y Zhou, 2011) y para identificar los aspectos que desencadenan la visita a los museos (Yamada y Fu, 2012).

En el campo del deporte y la actividad física la TPB ha tenido aplicación en trabajos muy importantes y recientes como el desarrollado por French et al. (2005) en el Departamento de Salud Pública de la Universidad de Cambridge, Inglaterra, cuyo objetivo fue analizar las creencias y actitudes que llevan a tener la intención de incrementar la actividad física en adultos ingleses. Se encontró que los aspectos afectivos son fundamentales a la hora de tomar la decisión de aumentar el volumen de ejercicio semanal, ya que se valora mejor el disfrute de las actividades que los beneficios que traerán, tanto así que si el usuario no percibe un ambiente agradable es muy probable que disminuya la cantidad de actividad que realiza y vuelva al sedentarismo.

Posteriormente, en el trabajo desarrollado por Anshel (2007), se analiza cada uno de los componentes del TPB y se relacionan con la actividad deportiva, en este caso, la actitud hacia el ejercicio refleja las creencias que tiene cada 
persona sobre los beneficios y consecuencias de realizarlo con regularidad, la norma subjetiva es la presión social que influye en la decisión de practicar ejercicio e incluso cómo afecta la permanencia en los programas, el control del comportamiento percibido se configura como las expectativas de las personas que desean ingresar a programas deportivos, o que ya están en ellos, e influye en la decisión de iniciar o mantenerse en dichas actividades, ya que, identificar que se cuenta con la habilidad de llegar a los objetivos propuestos es un factor determinante para la adherencia ${ }^{1}$; es más, el razonamiento finaliza relacionando estos factores con un suceso común observado en los deportistas profesionales retirados: "esta teoría podría explicar la decisión de muchos atletas de interrumpir los hábitos de ejercicio, cuando su carrera deportiva termina" (Anshel, 2007, p.10), debido a que ya no sienten la capacidad de competir y vencer.

Igualmente, Anderson y Lavallee (2008) utilizaron la TPB para analizar los factores que desencadenan la permanencia de los usuarios en los programas de entrenamiento deportivo, concluyendo que la TPB y la TRA ofrecen marcos teóricos pertinentes para examinar la adhesión a regímenes de entrenamiento nuevos, y además puede convertirse en una herramienta para orientar las estrategias que apunten a la constancia en las actividades (Anderson y Lavallee, 2008).

En el contexto local se han encontrado trabajos que intentan comprender los hábitos y preferencias en deporte y recreación en la ciudad de Medellín. Valencia, Tobón y Bedoya (2011), por ejemplo, realizan un análisis detallado de los factores que intervienen en la decisión de hacer o no actividad física o participar en programas de recreación, y de esta manera entregan información muy puntual sobre las predilecciones por estrato socioeconómico, género, estado civil y objetivos concretos de los participantes de los programas.

Finalmente, Colorado y Tavera (2015) desarrollaron una investigación denominada "Lealtad a la práctica deportiva", en la cual se concluye que "el modelo del TPB es adecuado para revelar el comportamiento de los deportistas y específicamente para explicar la lealtad a los programas deportivos ofertados por las instituciones públicas" (Colorado y Tavera, 2015, p.37); la investigación además genera propuestas para los gestores de este tipo de instituciones.

\footnotetext{
"Aproximadamente el 50\% de los individuos que se unen a los clubes de fitness abandonan el plazo de seis meses debido a su sentimiento de ningún progreso o falta de habilidad en la ejecución del programa de ejercicios" (Marcus, Bock, Pinto, y Clark, 1996, citados en Anshel, 2007, p.10).
} 


\section{Contexto colorado, A}

De ahí que la presente investigación aborde un tema que a lo largo del tiempo ha ido adquiriendo mayor relevancia no solo en el sentir popular, sino en la dinámica económica de los países:

El deporte y la recreación se han convertido en actividades fundamentales para los individuos y la sociedad en su conjunto. Estas tienen un fuerte impacto sobre la salud física y psicosocial de las personas, a la vez que repercuten en la calidad de vida, la productividad y la sana convivencia de las comunidades (Valencia, Tobón y Bedoya, 2011, p.11).

Por tal motivo, es tarea del campo de la administración que analiza el comportamiento del consumidor, develar las variables que influyen en los consumidores para desarrollar un comportamiento de lealtad hacia los servicios y programas deportivos que ofrecen tanto entidades públicas como privadas, con el fin de enfocar las estrategias que puedan llevar al aumento de la demanda.

Para comprender estos comportamientos definidos como "el manifiesto, la respuesta observable en una situación determinada con respecto a un objetivo determinado" (Ajzen, 2010), se propone la Teoría del Comportamiento Planificado por su comprobada utilidad para el análisis de los factores que desencadenan comportamientos relacionados con el deporte y la actividad física, entre ellos la lealtad (Colorado y Tavera, 2015).

\section{Teoría del Comportamiento Planificado}

La Teoría del Comportamiento Planificado (TPB) propuesta por Schifter y Ajzen (1985), derivada de la Teoría de la Acción Razonada (TRA, por sus siglas en inglés) (Ajzen y Fishbein, 1970), propone la intención como el mejor predictor del comportamiento y estaría influenciada por la actitud, la norma subjetiva y el control del comportamiento percibido (Schifter y Ajzen, 1985). Para la TPB la intención es el mejor determinante del comportamiento, pero la actitud, la norma subjetiva y el control percibido son los que facilitan la comprensión de los factores que explican las acciones del individuo (Rao y Troshani, 2007). La Figura 1 presenta la relación entre las variables del modelo TPB. 


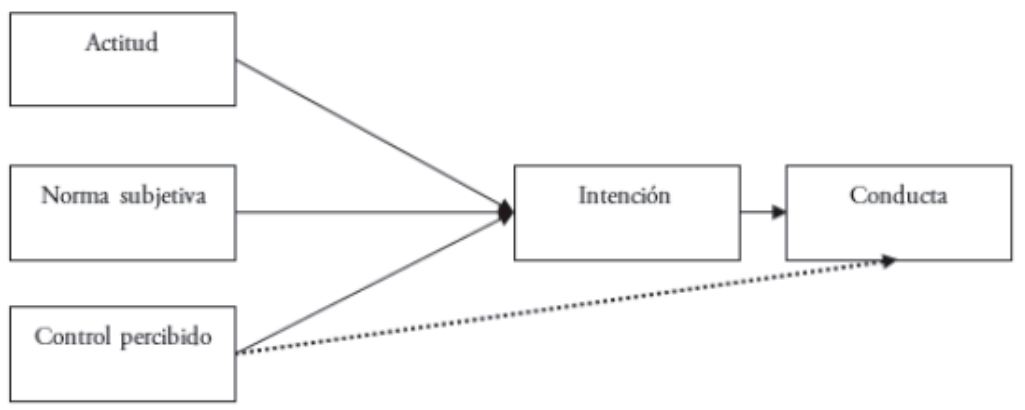

Figura 1. Teoría del comportamiento planificado.

Schifter y Ajzen (1985).

La actitud puede definirse de manera general como los sentimientos positivos o negativos de la persona sobre la realización de una conducta (Ajzen, 2010), y específicamente en el caso de servicios deportivos para niños, serían tenidos en cuenta para la creación de la actitud de los padres de familia frente a la información publicada por el centro deportivo sobre los beneficios del programa.

La norma subjetiva, por su parte, es la presión social percibida para participar o no participar en una conducta (Ajzen, 2010), de ahí que el grupo de sujetos cercanos asuma un papel importante a la hora de influir en las personas para tomar la decisión de inscribir a su hijo a un programa deportivo específico, acá adquieren gran relevancia los comentarios de familiares y vecinos sobre la alternativa que se vislumbra.

El control del comportamiento percibido se define como la percepción que el individuo tiene de la presencia o no de recursos $\mathrm{u}$ oportunidades necesarios para desarrollar el comportamiento en cuestión (Schifter y Ajzen, 1985), por lo cual, horarios, días y lugares para el desarrollo de las actividades y los costos asociados a ellas pueden influir en este aspecto.

\section{Modelo de hipótesis establecidas}

La literatura abordada permite suponer que la TPB es un modelo válido para explicar desencadenantes de la lealtad de los padres de familia hacia un programa deportivo para sus hijos, por tal motivo se establecen las siguientes hipótesis:

H1: La Actitud influye sobre la Intención de pertenecer a una escuela de iniciación deportiva. 


\section{Contexto colorado, A}

H2: La Norma Subjetiva influye sobre la Intención de pertenecer a una escuela de iniciación deportiva.

H3: El Control del Comportamiento Percibido influye sobre la Intención de pertenecer a una escuela de iniciación deportiva.

H4: El Control del Comportamiento Percibido influye sobre la Lealtad hacia una escuela de iniciación deportiva.

H5: La Intención influye sobre la Lealtad hacia una escuela de iniciación deportiva.

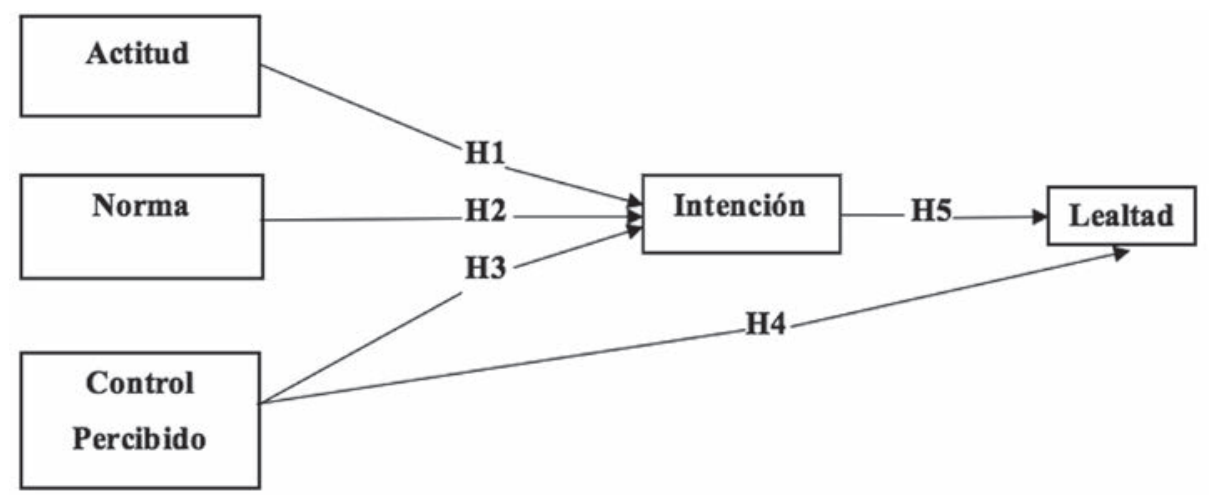

Figura 1. Modelo de hipótesis propuestas.

Elaboración propia.

\section{Metodología}

Se llevó a cabo una investigación de corte descriptivo, transversal simple, guiada bajo el método hipotético-deductivo. Teniendo en cuenta los planteamientos de Ajzen (2011), las hipótesis fueron contrastadas mediante una encuesta estructurada (Ajzen, 2006) que se realizó a una muestra de los 141 padres de familia que tenían inscritos sus hijos en una escuela de iniciación deportiva durante el año 2018.

La Tabla 1 recoge los ítems que fueron utilizados en cada escala así como su procedencia. La selección de dichos ítems fue revisada previamente con expertos, garantizando la validez de contenido. 
Tabla 1.

Escalas de medida utilizadas

\begin{tabular}{llc}
\hline Constructo & \multicolumn{1}{c}{ Escala } & \multicolumn{1}{c}{ Fuente } \\
\hline & $\begin{array}{l}\text { I.1.Tengo la intención de participar activamente en esta } \\
\text { escuela de iniciación deportiva durante los próximos } 6 \\
\text { meses }\end{array}$ & \\
\cline { 2 - 3 } Intención & $\begin{array}{l}\text { I.2. Voy a tratar de participar en las actividades deportivas } \\
\text { de esta escuela de iniciación deportiva durante los próximos }\end{array}$ & \\
& 6 Ajesen (2006) & \\
\cline { 2 - 3 } & $\begin{array}{l}\text { I.3. Planeo participar activamente en las actividades } \\
\text { deportivas de esta escuela de iniciación deportiva durante } \\
\text { los próximos 6 meses }\end{array}$ \\
\hline
\end{tabular}

A.1. Pertenecer a esta escuela de iniciación deportiva es para mí beneficioso

A.2. Pertenecer a esta escuela de iniciación deportiva es para mí placentero

Actitud A.3. Pertenecer a esta escuela de iniciación deportiva es para mí bueno

Ajzen (2006)

A.4. Pertenecer a esta escuela de iniciación deportiva es para mí valioso

A.5. Pertenecer a esta escuela de iniciación deportiva es para mí agradable

N.S.1. Las personas que son importantes para mí piensan que debo asistir regularmente a esta escuela de iniciación deportiva

N.S.2. Las personas cercanas a mí piensan que es probable

Norma que asista regularmente a esta escuela de iniciación deportiva

Subjetiva

N.S.3. Las personas cuyas opiniones valoro aprueban que

Ajzen (2006) asista regularmente a esta escuela de iniciación deportiva

N.S.4. Las personas importantes para mí valoran que haga parte de esta escuela de iniciación deportiva

C.P.1. Para mí es posible participar regularmente en esta escuela de iniciación deportiva

Control del C.P.2. Si quisiera, podría participar regularmente en esta

Comporta- escuela de iniciación deportiva

miento

C.P.3. Tengo control total para asistir regularmente a esta

Ajzen (2006)

Percibido escuela de iniciación deportiva

C.P.4. Depende de mí asistir regularmente a esta escuela de iniciación deportiva 


\section{Contexto colorado, A.}

\begin{tabular}{|c|c|c|}
\hline Constructo & Escala & Fuente \\
\hline \multirow{4}{*}{$\begin{array}{l}\text { Loyalty } \\
\text { Active }\end{array}$} & $\begin{array}{l}\text { L.A.1. Yo elegiría siempre pertenecer a esta escuela de } \\
\text { iniciación deportiva antes que a otros programas iguales }\end{array}$ & \multirow{4}{*}{$\begin{array}{c}\text { Zeithaml, } \\
\text { Berry y } \\
\text { Parasuraman } \\
\text { (1996) citados } \\
\text { en Bruner, } \\
\text { Hensel y } \\
\text { James (2005) }\end{array}$} \\
\hline & $\begin{array}{l}\text { L.A.2. Yo me consideraría un usuario fiel de los servicios } \\
\text { que ofrece esta escuela de iniciación deportiva }\end{array}$ & \\
\hline & $\begin{array}{l}\text { L.A.3. Estoy seguro de que durante los próximos } 6 \text { meses } \\
\text { seguiré asistiendo a los entrenamientos de esta escuela de } \\
\text { iniciación deportiva }\end{array}$ & \\
\hline & $\begin{array}{l}\text { L.A.4. Recomendaría a familiares y amigos que hagan parte } \\
\text { de esta escuela de iniciación deportiva }\end{array}$ & \\
\hline
\end{tabular}

Elaboración propia.

Se verificó la fiabilidad y validez mediante un análisis factorial confirmatorio utilizando el método de verosimilitud robusto, el cual es ideal para muestras con cierta anormalidad en los datos (Joreskog y Sorbom, 1990).

En el estudio de la validez convergente se verificó que todas las cargas factoriales estandarizadas de los indicadores fueran muy cercanas o superiores a 0,6 (Bagozzi \& Yi, 1988), que el promedio de cargas factoriales estandarizadas fuera muy cercano o superior a 0,7 , que el test de multiplicadores de Lagrange no propusiera relaciones adicionales a las planteadas (Hatcher, 1994), y que existiera un buen ajuste del modelo. El proceso exigió la depuración de los ítems $\mathrm{A} 3, \mathrm{~A} 5$ y CP4, tras lo cual fue posible garantizar las condiciones anteriores con un buen ajuste del modelo, tal como lo evidencia la Tabla 2 .

Tabla 2.

Análisis de la validez convergente

\begin{tabular}{|c|c|c|c|c|}
\hline Constructo & Ítem & $\begin{array}{l}\text { Cargas Factoriales } \\
\text { Estandarizadas }\end{array}$ & $\begin{array}{c}\text { Promedio Cargas } \\
\text { Factoriales } \\
\text { Estandarizadas }\end{array}$ & $\begin{array}{l}\text { Valor } \mathrm{t} \\
\text { robusto }\end{array}$ \\
\hline \multirow{3}{*}{ Intención de uso (I) } & IN1 & 0,785 & \multirow{3}{*}{0,864} & 5,29 \\
\hline & IN2 & 0,943 & & 7,38 \\
\hline & IN3 & 0,863 & & 7,06 \\
\hline \multirow{3}{*}{$\begin{array}{c}\text { Actitud hacia la práctica } \\
\text { deportiva (A) }\end{array}$} & $\mathrm{ACl}$ & 0,913 & \multirow{3}{*}{0,885} & 8,13 \\
\hline & $\mathrm{AC} 2$ & 0,969 & & 7,80 \\
\hline & $\mathrm{AC} 4$ & 0,772 & & 7,22 \\
\hline
\end{tabular}




\begin{tabular}{|c|c|c|c|c|}
\hline Constructo & Ítem & $\begin{array}{l}\text { Cargas Factoriales } \\
\text { Estandarizadas }\end{array}$ & $\begin{array}{l}\text { Promedio Cargas } \\
\text { Factoriales } \\
\text { Estandarizadas }\end{array}$ & $\begin{array}{l}\text { Valor t } \\
\text { robusto }\end{array}$ \\
\hline \multirow{4}{*}{ Norma subjetiva (NS) } & NS1 & 0,881 & \multirow{4}{*}{0,808} & 7,00 \\
\hline & NS2 & 0,653 & & 6,31 \\
\hline & NS3 & 0,787 & & 6,19 \\
\hline & NS4 & 0,910 & & 5,47 \\
\hline \multirow{3}{*}{$\begin{array}{l}\text { Control percibido del } \\
\text { comportamiento (CP) }\end{array}$} & $\mathrm{CP} 1$ & 0,774 & \multirow{3}{*}{0,783} & 8,96 \\
\hline & $\mathrm{CP} 2$ & 0,840 & & 7,90 \\
\hline & CP3 & 0,736 & & 7,82 \\
\hline \multirow{4}{*}{ Lealtad (LA) } & LA1 & 0,698 & \multirow{4}{*}{0,755} & 8,28 \\
\hline & LA2 & 0,751 & & 8,73 \\
\hline & LA3 & 0,801 & & 7,54 \\
\hline & LA4 & 0,768 & & 5,06 \\
\hline
\end{tabular}

S-B Chi cuadrado $($ g.l. $=109)=137,12(\mathrm{p}<0,01)$; BBNFI $=0,801$; BBNNFI $=0,937$; CFI $=$ 0,$949 ;$ IFI $=0,952 ;$ RMSEA $=0,043(0,012-0,064)$

Elaboración propia.

Se procedió a verificar la validez discriminante (ver Tabla 3) evidenciando que el valor 1 no estuviera contenido en el intervalo de confianza de las correlaciones entre las diferentes escalas (Anderson y Gerbing, 1988), y que el Índice de Varianza Extraída (IVE) de cada constructo sobrepasara el cuadrado de las covarianzas entre cada par de factores (Fornell y Larcker, 1981).

Tabla 3.

Análisis de la validez discriminante

\begin{tabular}{cccccc}
\hline & I & A & NS & CP & LA \\
\hline I & 0,866 & 0,754 & 0,556 & 0,643 & 0,708 \\
\hline $\mathrm{A}$ & {$[0,5991 ; 0,9088]$} & 0,889 & 0,630 & 0,726 & 0,697 \\
\hline $\mathrm{NS}$ & {$[0,2914 ; 0,8206]$} & {$[0,37716 ; 0,88284]$} & 0,814 & 0,679 & 0,718 \\
\hline $\mathrm{CP}$ & {$[0,4372 ; 0,8488]$} & {$[0,57312 ; 0,87888]$} & {$[0,39284 ; 0,96516]$} & 0,787 & 0,730 \\
\hline $\mathrm{LA}$ & {$[0,5257 ; 0,8902]$} & {$[0,56568 ; 0,82832]$} & {$[0,58864 ; 0,84736]$} & {$[0,57712 ; 0,88288]$} & 0,755 \\
\hline **p<,01; *p<,05. Debajo de la diagonal: intervalo de confianza para la correlación entre factores. Diago- \\
nal: raíz cuadrada de la varianza extraída. Sobre la diagonal: correlación estimada entre los factores.
\end{tabular}

Elaboración propia. 


\section{Contexto colorado, A}

Para determinar la fiabilidad del instrumento utilizado, se calcularon tres indicadores diferentes de la fiabilidad de las escalas con miras a garantizarla:

- El Alfa de Cronbach, cuyo valor de referencia lo recomiendan Churchill (1979) y Nunnally y Bernstein (1994) como mayor a 0,7.

- El Índice de Fiabilidad Compuesta (IFC), cuyo valor de referencia es estimado por Fornell y Larcker (1981) como cercano o mayor a 0,7.

- El Índice de Varianza Extraída (IVE), que Fornell y Larcker (1981) estiman positivo un valor cercano o mayor a 0,5 .

Como se observa en la Tabla 4, todos los constructos cumplieron satisfactoriamente dos de los tres indicadores y el $60 \%$ de los constructos sobrepasaron los tres indicadores, se asume una muy buena fiabilidad del modelo de medida.

Tabla 4.

Análisis de fiabilidad, fiabilidad compuesta y varianza extraídas de las escalas de medida

\begin{tabular}{lccc}
\hline \multicolumn{1}{c}{ Constructos } & $\begin{array}{c}\text { Alfa de } \\
\text { Cronbach }\end{array}$ & $\begin{array}{c}\text { Índice de Fiabilidad } \\
\text { Compuesta (IFC) }\end{array}$ & $\begin{array}{c}\text { Índice de Varianza } \\
\text { Extraída (IVE) }\end{array}$ \\
\hline Intención de Uso (I) & 0,896 & 0,776 & 0,750 \\
\hline Actitud (A) & 0,909 & 0,808 & 0,790 \\
\hline Norma subjetiva (NS) & 0,866 & 0,705 & 0,663 \\
\hline Control Percibido (CP) & 0,814 & 0,671 & 0,615 \\
\hline Lealtad (LA) & 0,830 & 0,637 & 0,571 \\
\hline
\end{tabular}

Elaboración propia.

\section{Resultados}

Una vez garantizadas la fiabilidad y la validez del instrumento, se contrastaron las hipótesis mediante ecuaciones estructurales, utilizando para ello el método de máxima verosimilitud robusto.

Los resultados obtenidos evidenciaron buenos indicadores de ajuste y la aceptación de la mayoría de las hipótesis planteadas, tal como lo evidencia la Tabla 5 y lo ilustra la Figura 3. 
Tabla 5.

Contraste de hipótesis

\begin{tabular}{|c|c|c|c|c|}
\hline \multicolumn{2}{|r|}{ Hipótesis } & $\begin{array}{l}\text { Coeficiente } \\
\text { Estandarizado }\end{array}$ & $\begin{array}{l}\text { Valor T } \\
\text { Robusto }\end{array}$ & \multirow{2}{*}{$\begin{array}{l}\text { Conclusión } \\
\text { No rechazada }\end{array}$} \\
\hline $\mathrm{H} 1$ & $\begin{array}{l}\text { La Actitud influye sobre la Intención de } \\
\text { pertenecer a una escuela de iniciación deportiva }\end{array}$ & 0,595 & $3,01 * * *$ & \\
\hline $\mathrm{H} 2$ & $\begin{array}{l}\text { La Norma Subjetiva influye sobre la } \\
\text { Intención de pertenecer a una escuela de } \\
\text { iniciación deportiva }\end{array}$ & N.S. & N.S. & Rechazada \\
\hline $\mathrm{H} 3$ & $\begin{array}{l}\text { El Control del Comportamiento Percibido } \\
\text { influye sobre la Intención de pertenecer a } \\
\text { una escuela de iniciación deportiva }\end{array}$ & N.S. & N.S. & Rechazada \\
\hline $\mathrm{H} 4$ & $\begin{array}{l}\text { El Control del Comportamiento } \\
\text { Percibido influye sobre la Lealtad hacia } \\
\text { una escuela de iniciación deportiva. }\end{array}$ & 0,528 & $3,34 *, * *$ & No rechazada \\
\hline H5 & $\begin{array}{l}\text { La Intención influye sobre la Lealtad } \\
\text { hacia una escuela de iniciación deportiva. }\end{array}$ & 0,377 & $2,32 * * *$ & No rechazada \\
\hline \multicolumn{5}{|c|}{$\begin{array}{c}\text { S-B Chi cuadrado }(\text { g.l. }=111)=139,57(p<0,01) ; \text { BBNFI }=0,801 ; \text { BBNNFI }=0,937 ; \text { CFI }= \\
0,949 ; \text { IFI }=0,952 ; \text { RMSEA }=0,043(0,012-0,064)\end{array}$} \\
\hline \multicolumn{5}{|c|}{$* \mathrm{p}<0.1 ; * * \mathrm{p}<0.05$} \\
\hline
\end{tabular}

Elaboración propia.

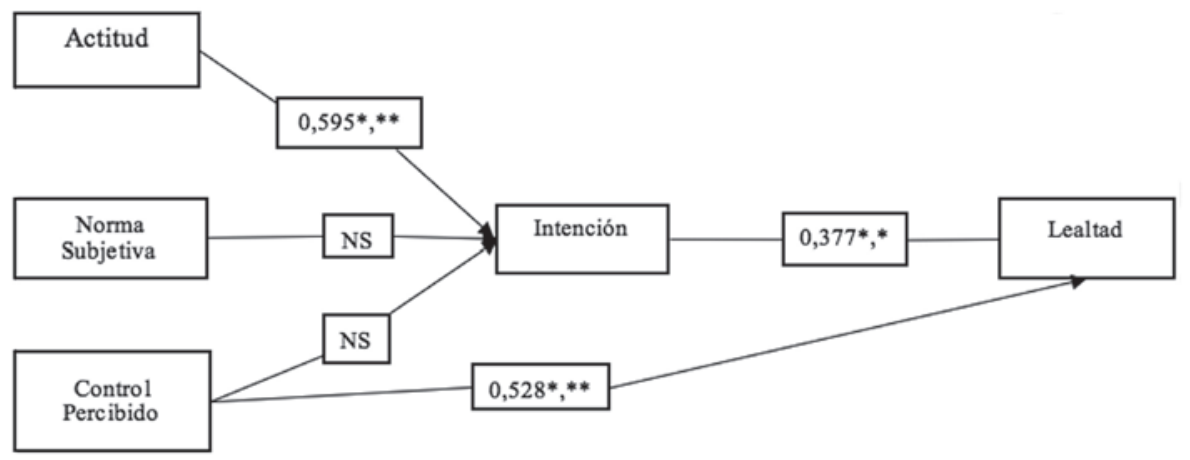

Figura 3. Modelo final contrastado.

Elaboración propia. 


\section{Contexto colorado, A}

La Figura 3 permite visualizar los resultados del contraste empírico del modelo. En el caso de la escuela de iniciación deportiva analizada, se evidencia que la Actitud y el Control del Comportamiento Percibido se constituyen en los desencadenantes más importantes para desarrollar la intención de participar en dicha institución en primera instancia y posteriormente para desarrollar lealtad hacia ésta. A su vez, al observar los efectos directos e indirectos de los factores que inciden en el desarrollo de la lealtad, el constructo que mayor influencia ejerce es el Control del Comportamiento Percibido.

\section{Conclusiones}

Los resultados demuestran que para este caso la actitud es el principal influenciador de la intención para desarrollar la lealtad a una escuela de iniciación deportiva, ya que las cargas factoriales de la norma subjetiva y el control percibido fueron demasiado bajas y por lo tanto no significativas.

Al analizar los aspectos que constituyen la actitud se encuentran percepciones como beneficioso, placentero, bueno, valioso y agradable, que solo pueden desarrollarse a través de la manifestación de declaraciones concretas sobre los beneficios que entregará el proceso, luego se validan los resultados a través de encuestas de satisfacción y grupos focales para identificar el nivel de aceptación de la propuesta y, finalmente, la conexión con el cliente por medio de experiencias significativas para ellos y sus hijos.

Por otro lado, se encuentra que el Control del Comportamiento Percibido constituye la variable más importante para desencadenar el comportamiento de fidelización hacia la escuela de iniciación deportiva, dado que aspectos como flexibilidad de horarios, precios competitivos y escenarios deportivos con buena ubicación, hacen posible la participación de los padres de familia y sus hijos.

Para reafirmar lo anterior, se destaca como elementos fundamentales de la escuela de iniciación deportiva que los días y horarios de entrenamiento no compiten con las actividades laborales de los clientes, sus costos son proporcionados para el perfil de los padres de familia y las condiciones logísticas como parqueaderos, unidades sanitarias y cafeterías generan un ambiente agradable y placentero.

Igualmente, el control del comportamiento percibido se evidencia en la capacidad de alcanzar los logros y en este factor los padres de familia pueden realizar un seguimiento detallado, ya que la institución entrega periódicamente reportes sobre el proceso individual y además genera reportes de investigación 
sobre los avances de los niños participantes, con lo cual marca gran diferencia frente a otros servicios deportivos similares.

Finalmente, puede concluirse que el modelo TPB es una buena herramienta para explicar el comportamiento de lealtad hacia una escuela de iniciación deportiva, ya que los usuarios manifestaron que las actividades en las que participan tienen impacto en el desarrollo de su fidelización. Esto evidenciao que aunque es importante la actitud y la intención futura de participar, tiene mayor incidencia el control del comportamiento percibido.

\section{Referencias}

Ajzen, I. (1985). From intentions to actions: A theory of planned behavior. In J. Kuhl \& J. Beckman (Eds.), Action-control: From cognition to behavior (pp. 11-39). Heidelberg: Springer.

Ajzen, I. (2006). Constructing a TPB questionnaire: Conceptual and methodological considerations. Recuperado de http://www.uni-bielefeld. de/ikg/zick/ajzen $\% 20$ construction $\% 20 \mathrm{a} \% 20$ tpb\%20questionnaire.pdf [Consultado el 10 de mayo de 2012].

Ajzen, I. (2011). Theory of Planned Behaviour: Reactions and Reflections. Psychology \& Health, 26(9), 1113-1127.

Ajzen, I. \& Fishbein, M. (1970). The prediction of behavior from attitudinal and normative variables. Journal of Experimental Social Psychology, 6(4), 466-487.

Anderson, A. \& Lavallee, D. (2008). Applying the theories of Reasoned Action and Planned Behavior to athlete training adherence behavior. Applied Psychology: An International Review, 57(2), 304-312.

Anderson, J. \& Gerbing, D. (1988). Structural Equation Modeling in Practice: A review and recommended two-step approach. Psychological Bulletin, 103, 411-423.

Anshel, M. (2007). Conceptualizing Applied Exercise Psychology. The Journal of the American Board of Sport Psychology, 1, 1-44.

Bagozzi, R. \& Yi, Y. (1988). On the evaluation of structural equation models. Journal of Academy of Marketing Science, 16, 74-94. 


\section{Contexto colorado, A.}

Bruner, G., Hensel, P. \& James, K. (2005). Marketing Scales Handbook. Vol. 4.: A Compilation of Multi-Item Measures for Consumer Behavior \& Advertising. Thomson Higher Education.

Churchill Jr, G. (1979). A paradigm for developing better measures of marketing constructs. Journal of Marketing Research, 16(1), 64-73.

Colorado, A. y Tavera, J. (2015). Lealtad a la práctica deportiva: Una aplicación de la TPB a los servicios públicos para la práctica deportiva. Sportk: Revista Euroamericana de Ciencias del Deporte, 4(1), 29-40.

Consejo Superior de Deportes de España. (2005). Encuesta de hábitos deportivos. Encuestas procesadas en el estudio de Hábitos Deportivos de los Españoles en 2005. Consejo Superior de Deportes de España. Recuperado de http:// www.csd.gob.es/csd/sociedad/encuesta-de-habitos-deportivos/encuestade-habitos-deportivos-2005/9-el-deporte-como-consumo-y-espectaculode-masas.

Della, L., DeJoy, D. \& Lance, C. (2008). Promoting fruit and vegetable consumption in different lifestyle groups: Recommendations for program development based on behavioral research and consumer media data. Health Marketing Quarterly, 25(0), 66-96.

Ferdous, A. (2010). Applying the Theory of Planned Behavior to Explain marketing managers' perspectives on sustainable marketing. Journal of International Consumer Marketing, 22(4), 313-325.

Fornell, C. \& Larcker, D. (1981). Evaluating structural equation models with unobservable variables and measurement error. Journal of Marketing Research, 18, 39-50.

French, D., Sutton, S., Hennings, S., Mitchell, J., Wareham, N., Griffin, S. et al. (2005). The importance of affective beliefs and attitudes in the Theory of Planned Behavior: Predicting intention to increase physical activity. Journal of Applied Social Psychology, 35(9), 1824-1848.

Hatcher, L. (1994). A step-by-step approach to using the SAS system for factor analysis and structural equation modeling. SAS Publishing.

Joreskog, K. \& Sorbom, D. (1990). Analysis of linear structural relationships by the method of maximum likelihood: User's guide. Mooresville, IN.

Lynne, G. \& Casey, C. (1995). Conservation technology adoption decisions and the theory of planned behavior. Journal of Economic Psychology, 16(4), 581-598. 
Naciones Unidas. (2005). Concepto: Educación, salud, desarrollo y Paz.

Nunnally, J. \& Bernstein, I. (1994). Psychometric Theory. $3^{\text {rd }}$ ed. New York: McGraw-Hill.

Rao, S. \& Troshani, I. (2007). A conceptual framework and propositions for the acceptance of mobile services. Journal of Theoretical and Applied Electronic Commerce Research, 2(2), 61-73.

Schifter, D. \& Ajzen, I. (1985). Intention, perceived control, and weight loss: An application of the theory of planned behavior. Journal of Personality and Social Psychology, 49(3), 843-851.

Valencia, G., Tobón, D. y Bedoya, J. (2011). Hábitos y preferencias por recreación y deporte en Medellín: Una aplicación de modelos logísticos. Lecturas de Economía, 74, 9-35.

Yamada, N. \& Fu, Y.-Y. (2012). Using the Theory of Planned Behavior to identify beliefs underlying visiting the Indiana State Museum. Journal of Travel \& Tourism Marketing, 29(2), 119-132.

Yang, H. \& Zhou, L. (2011). Extending TPB and TAM to mobile viral marketing: An exploratory study on American young consumers' mobile viral marketing attitude, intent and behavior. Journal of Targeting, Measurement \& Analysis for Marketing, 19(2), 85-98.

\section{Para citar este artículo:}

Colorado, A. (2019). Desencadenantes de la lealtad de padres de familia hacia un programa deportivo para sus hijos. Avances en el campo de las gestión deportiva. En-Contexto, 7(11), 165-181. 